\title{
Traffic Management of Multimedia over ATM Networks
}

\author{
Bing Zheng and Mohammed Atiquzzaman, University of Dayton
}

\begin{abstract}
Multimedia is characterized by bursty traffic and often stringent real-time constraints. Multimedia traffic requires quality of service guarantees. This gives rise to traffic management issues in order to obtain high network utilization and quality of service guarantee to the multimedia stream. The aim of this article is to present an overview of the traffic management issues and their possible solutions for transmission of multimedia over asynchronous transfer mode networks.
\end{abstract}

M ultimedia consists of voice, video, and data in the same application. When multimedia is transmitted over a network, video traffic consumes most of the bandwidth, and also requires a high level of quality of service $(\mathrm{Q} O S)$ guarantee from the network. In order to reduce the bandwidth requirement, video is therefore compressed before transmission over the network. V ideo compression is based on removing the temporal and spatial redundancy of information in a video stream.

A n M PEG compressed video consists of different types of frames $(I, B$, and $P)$, of varying bit rates. A number of these frames are grouped together to form a group of pictures (GoP). A G oP begins with an I-frame, followed by B-and Pframes. If a G oP includes $n$ frames with $(m-1)$ B-frames between consecutive P-frames, the GoP is denoted $\mathrm{MmNn}$. Compression greatly reduces the bit rate of the multimedia stream. However, compressed video is highly bursty in nature. The bit rate changes abruptly from frame to frame; the Iframe has the highest bit rate among the three frame types, and the B-frame usually has the smallest rate. The bursty nature of the multimedia stream necessitates managing the traffic efficiently when transmitted over an asynchronous transfer mode (A TM ) network. This gives rise to a number of traffic management issues related to transmission of multimedia traffic over ATM .

A dvantages of ATM networks, such as high trunk speed, low bit error rate, flexible service type (bandwidth on demand), and high multiplexing capacity make it very suitable for transporting multimedia with Q OS guarantees. Statistical multiplexing of bursty video sources in an A TM network improves the bandwidth utilization of the network and lowers the service cost. F urthermore, the multiplexing of multimedia streams reduces the burstiness and bandwidth requirement of the aggregate traffic. H owever, coincidence of the peaks of many video streams may result in congestion in the network and degrade the quality of the video. Congestion control is important to guarantee the quality of multimedia. M oreover, in multiplexed transmission, efficient bandwidth allocation among the multiple multimedia streams will improve network resource utilization. A network can be managed much more efficiently if the traffic entering the network is smooth. For transmitting video, the bursty multimedia stream can be shaped to smooth out the multimedia traffic stream. Flow control is used to regulate the traffic rate between the sender and the receiver (e.g., a client/server type of system) so that a fast/slow transmitter will not result in overflow/under-

\begin{tabular}{|c|c|c|c|c|c|c|}
\hline $\begin{array}{l}\text { Service } \\
\text { type }\end{array}$ & $\begin{array}{l}\text { Parameters during } \\
\text { connection setup }\end{array}$ & $\begin{array}{l}\text { Bandwidth } \\
\text { renegotiation }\end{array}$ & $\begin{array}{l}\text { Bandwidth } \\
\text { guarantee }\end{array}$ & $\begin{array}{l}\text { QoS } \\
\text { guarantee }\end{array}$ & $\begin{array}{l}\text { Bandwidth } \\
\text { utillization }\end{array}$ & Cost \\
\hline CBR & PCR rate & No & Yes & Yes & Very low & Very high \\
\hline VBR & PCR, SCR & No & Yes & Yes & Low & High \\
\hline$A B R$ & $\begin{array}{l}\text { MCR, PCR, ICR, } \\
\text { TBE, RIF, RDF, etc. }\end{array}$ & Yes & Only MCR & Acceptable & Very high & Very low \\
\hline
\end{tabular}

Table 1. Characteristics of ATM services which are suitable for multimedia. 


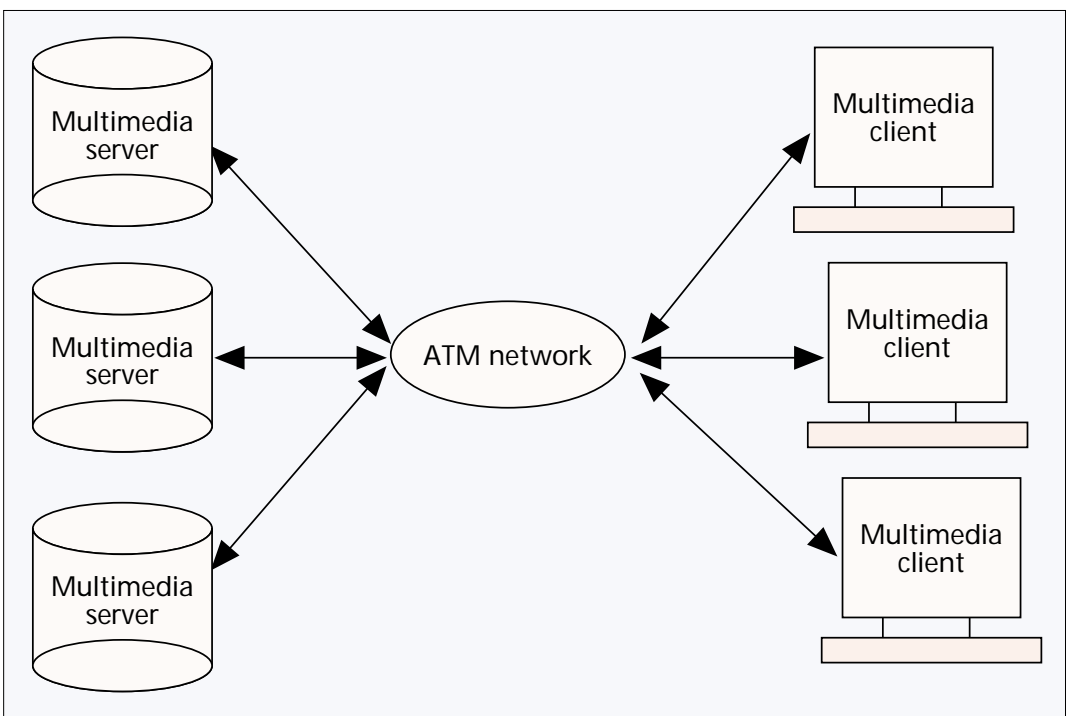

Figure 1. A model of a client/server-based multimedia application over ATM networks.

section, the basic concepts of the A TM network and its service categories are outlined. We then discuss the feedback technique used in traffic management. N ext, the commonly used traffic shaping and rate control techniques are discussed. B andwidth allocation in ATM networks is reviewed, followed by a review of congestion control schemes. F inally, flow control and scalable video transmission techniques are discussed, respectively.

\section{THE ATM NETWORK AND ITS Service CATEgories}

A TM F orum has defined and standardized five types of service: constant bit rate ( $C B R$ ), variable bit rate (VBR) including real-time $V B R$ ( $r t-V B R$ ) and non-real-time $V B R$ (nrt-VBR), available bit rate (ABR), and unspecified bit rate $(U B R$ ). $R$ eal-time multimedia transmission requires rate guarantees for an acceptable picture quality. Since U BR cannot guarantee the transmission bit rate, it is rarely used to transmit multimedia over an A TM network. Multimedia transmission therefore usually takes place over CBR, VBR, and $A B R$. To better understand the suitability of the services for multimedia transmission, the specifications and characteristics of the various traffic classes are shown in Table 1.

For a CBR connection, the peak cell rate (PCR) is negotiated during connection setup and is guaranteed by the network for the duration of the connection, resulting in high transmission quality and service. H owever, because of the bursty nature of compressed video, the PCR may not be fully utilized at all times, resulting in low bandwidth utilization and high service costs. VBR service negotiates the PCR and the sustainable cell rate ( $S C R$ ) during connection setup, both of which are guaranteed throughout the entire duration of the connection.

To improve the utilization of the network bandwidth, the A TM F orum has standardized A BR service, which requires a large number of parameters (as shown in Table 1 ) to be negotiated during connection setup. MCR is the minimum cell rate guaranteed by the network, and can be used to provide an acceptable Q oS for multimedia applications [1]. If there is bandwidth available in the network, the ABR service offers the possibility of flexible bandwidth renegotiation during transmission using resource management ( $R M$ ) cells. CBR and $V B R$ do not allow bandwidth renegotiation after a connection has been set up. The bandwidth available during a connection is determined by the PCR (in the case of CBR) or
$P C R$ and SCR (in the case of VBR) which have been negotiated at connection setup.

\section{FEEDBACK AND TRAFFIC MANAGEMENT}

Feedback can be successfully used to manage multimedia traffic over A TM -based networks. A feedback system usually consists of three components, as shown in Fig. 2. The sampler obtains information regarding the network. Such information may consist of the congestion level in the network, which may be obtained from the buffer occupancy and explicit rate information from the switches. The feedback information obtained from the sampler is used by the control algorithm to calculate the control parameters for the next step. In a client/server model, this task is done by the server to control the source rate. The control parameters could include the data rate of the multimedia source and the priority label of the cells belonging to the different frames of the video traffic. The execution unit implements the control scheme using the control parameters calculated by the control/logic unit. Typical execution units in A TM networks are admission/connection controller, rate controller, priority label assigner, and so on.

The task of traffic management can be classified into six components (as will be described in detail in the following sections), with feedback forming the core of the various components, as shown in Fig. 3. W e use solid lines to show the dependence between the components and feedback control, and dotted lines to show the internal relationship or similarities between the components. For example, traffic shaping may receive feedback from the network and may shape the traffic in order to control congestion in the network. On the contrary, flow control is an end-to-end behavior and may not have any relationship with the other components, such as congestion. Similarly, bandwidth allocation, scalable video transmission, and admission control contribute to control of congestion in the network; although the tasks of congestion control, bandwidth allocation, admission control, traffic shaping, and scalable transmission are different, their objectives are to help the network manage its multiplexed traffic and guarantee Q oS to the users.

Feedback is used by many traffic management functions, although the feedback information is used differently by the functions. For example, the closed-loop feedback of the ABR service obtains information about network congestion from the backward R M cells, whereas data packets may be used to

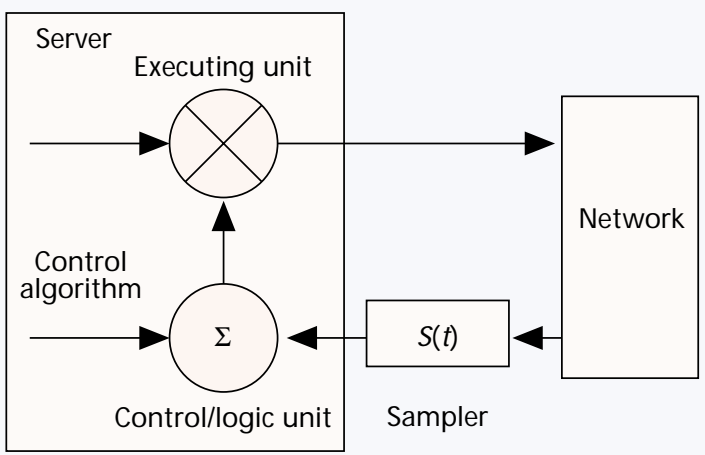

Figure 2. Components foR a feedback system. 


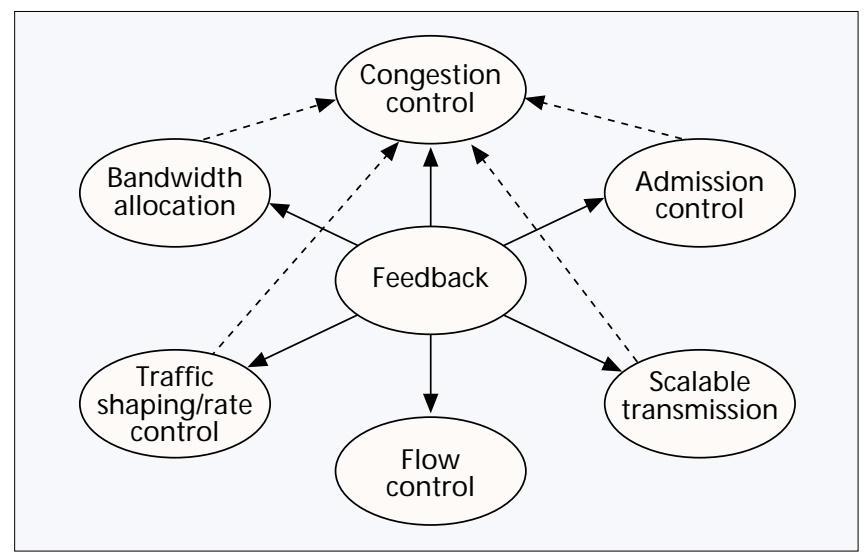

Figure 3. The relationship between feedback and various components of traffic management.

convey the congestion information to the server when the $\mathrm{VBR}$ service is used to carry multimedia traffic.

\section{Traffic Shaping/Rate Control}

The variable bit rate of the different frames of video traffic necessitates the shaping of the traffic before being injected into the network. Traffic shaping can be done either at the end systems, or in the network by the switching hardware.

\section{Traffic Shaping/Rate Control at End Systems}

Traffic shaping or rate control at the end systems can be implemented by the server using a L eaky Bucket (single or dual) shaper [2] consisting of a buffer and a rate controller, as shown in Fig. 4. The main issues are the rate control mechanism, shaper delay and delay variation, and the shaper buffer size at the server. The rate controller determines the outgoing data rate which should be consistent with the bandwidth available from the network. A n easy-to-implement set of traffic descriptors is therefore a key factor in obtaining good performance from the shaper. Closed-loop feedback rate control which utilizes feedback obtained from the network (previous section) can be used to control the traffic rate.

The shaper needs a large buffer for accumulating the incoming bursty multimedia stream. H owever, if the outgoing rate of the shaper is low, a large shaper buffer may result in long delay variation, thereby degrading the video quality at the client. Therefore, there exists a trade-off between the buffer size, shaper delay, and outgoing rate of the shaper. A solution to this problem is to set the shaper's outgoing rate equal to the average rate of the multimedia stream, and to dimension the shaper buffer size just large enough to store the small-scale fluctuations between the incoming and outgoing data rates of the shaper [2].

\section{Traffic Shaping by the Network}

If the multiplexed traffic inside the network is bursty, the network may implement traffic shaping to manage its resources. This may incur additional delay and delay variation inside the network, and has implications on the quality of the multimedia traffic received at the client. A direct consequence of network traffic shaping is the requirement of a large buffer at the client to smooth out the delay and jitter due to traffic shaping by the network.

\section{BANDWIDTH ALLOCATION}

The bursty nature of multimedia traffic enables multiplexing of a large number of sources, giving rise to the issue of efficiently allocating the network bandwidth among the sources. The two main issues are bandwidth sharing and dynamic bandwidth allocation.

\section{BANDWIDTH SHARING}

The bandwidth sharing method [3] relies on sharing the link bandwidth among a number of connections using one of the following two methods.

- Fair bandwidth sharing is based on sharing the link bandwidth $\mathrm{C}$ among $\mathrm{N}$ connections such that $\sum_{i=1}^{N} \mathrm{C}_{\mathrm{i}}(\mathrm{t}) \leq \mathrm{C}$ where $c_{i}(t)$ is the bandwidth assigned to connection i during connection setup.

- Bandwidth scheduling assigns a limited amount of bandwidth to a number of video connections according to specific scheduling time slots. This scheme is easy to implement, but suffers from lack of flexibility due to the time-varying nature of the bandwidth requirements of multimedia streams.

\section{Dynamic BandWidTh Renegotiation}

Dynamic bandwidth renegotiation is based on periodically renegotiating the bandwidth of a connection depending on its requirements. If $b(t)$ denotes the bandwidth allocated to a connection at time $t, \tau$ the time interval for bandwidth renegotiation, and $\mathrm{e}(\mathrm{t})$ and $\mathrm{g}(\mathrm{t})$ the rate and congestion information from the network, respectively, at time t, the dynamic bandwidth allocation for a connection can be expressed as $b(t)=$ $f(b(t-\tau), e(t), g(t))$. The following schemes can be used to implement dynamic bandwidth renegotiation.

Feedback-Based Dynamic Bandwidth Renegotiation Since an interactive multimedia stream is bursty at various timescales, its bandwidth requirement changes from time to time. To utilize the bandwidth efficiently, the bandwidth allocated to a multimedia stream needs to be dynamically renegotiated.

The ABR service offers a dynamic bandwidth renegotiation scheme throughout the duration of a connection using the $\mathrm{R} M$ cells (which carry feedback regarding the level of network congestion). A t A BR connection setup, the source (server) negotiates (with the network) a number of parameters such as those given in Table 1. H owever, the server can request renegotiation of allowable bandwidth at any instant of time by renegotiating the allowable cell rate (ACR) at any time throughout the duration of the connection using the explicit rate (ER) field of the $R M$ cell. The value of the $A C R$ allocated by the network lies between the MCR and PCR, and of course depends on the available network bandwidth.

Prediction-Based Dynamic Bandwidth Allocation - This scheme is especially suitable for real-time encoded multimedia transmission. The server looks at the characteristics of the multimedia traffic streams and anticipates their future bandwidth requirements [4]. B ased on the anticipated requirements, the server attempts to obtain additional bandwidth in advance so that the multimedia can be transmitted with minimum delay at the server.

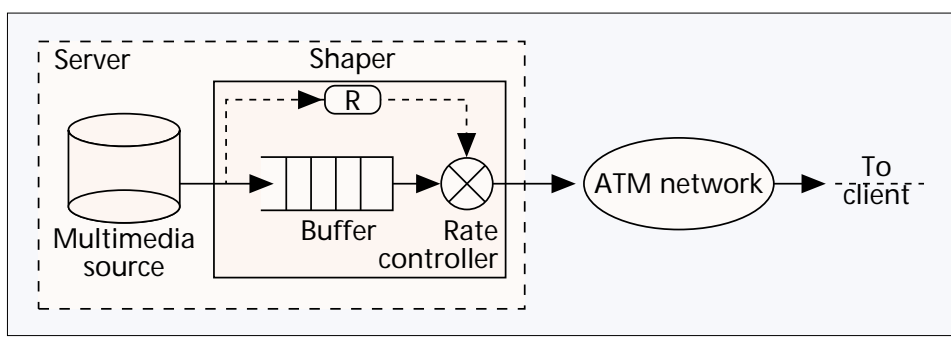

Figure 4. The shaper at the server. 
To summarize, the feedback-based technique is effective in dynamic bandwidth sharing among multiple multimedia streams. D ue to the trick modes in interactive multimedia, the bandwidth requirement varies over time. $H$ ence, $A B R$ service with the possibility of acquiring bandwidth throughout the duration of the connection is very suitable for dynamic bandwidth renegotiation.

\section{DyNAMiC BandWIDTH ReNegotiation IN INTERACTIVE MULTIMEDIA TRANSMISSION: AN EXAMPLE}

Once an ABR connection has been setup, the video source can dynamically request additional bandwidth using the $E R$ field of the RM cells. A n ABR service is therefore very suitable for transmitting interactive multimedia whose bandwidth requirement varies due to the use of trick modes. In this section we give an example of an interactive multimedia system using dynamic bandwidth renegotiation for management of traffic.

Figure 5 shows a model of an interactive client/serverbased video-on-demand system. In such a multimedia system [5], the client can perform trick modes such as stop/pause, playback, fastforward, and fastbackward, as shown in Fig. 6. Therefore, the client can be modeled as being in one of the states (play, pause, etc.). The long-term behavior of the client can be described by a steady state probability vector $\mathbf{S}=\left(\mathrm{S}_{0}\right.$, $\mathrm{S}_{1}, \mathrm{~S}_{2}, \mathrm{~S}_{3}$ ) which can be obtained by solving the steady vector equation $\mathbf{S}=\mathbf{S} \mathbf{P} . \mathrm{S}_{0}, \mathrm{~S}_{1}, \mathrm{~S}_{2}$, and $\mathbf{S}_{3}$ correspond to the stop/pause, normal playback, fastforward, and fastbackward states, and $\mathbf{P}$ is the transition matrix of client states [5] as shown in Fig. 6.

If the bit rate of multimedia during playback is $\lambda_{1}(t)$, and the bit rate during fastforward and fastbackward (F FW AND FBW ) is k times that of playback, the expected bit rate (or bandwidth requirement) as a function of the level of client interactivity is given by $E[\lambda(t)]=\left[S_{1}+k\left(S_{2}+\right.\right.$ $\left.\left.\mathrm{S}_{3}\right)\right] \lambda_{1}(\mathrm{t})$. The level of client interactivity (described by the F FW /FBW probability of a client) determines the state probabilities of a client.

Because the rate at which the server buffer (Fig. 5) transmits multimedia into the network depends on the level of network congestion, the server buffer occupancy can be used as a network congestion indicator. To avoid buffer overflow, we

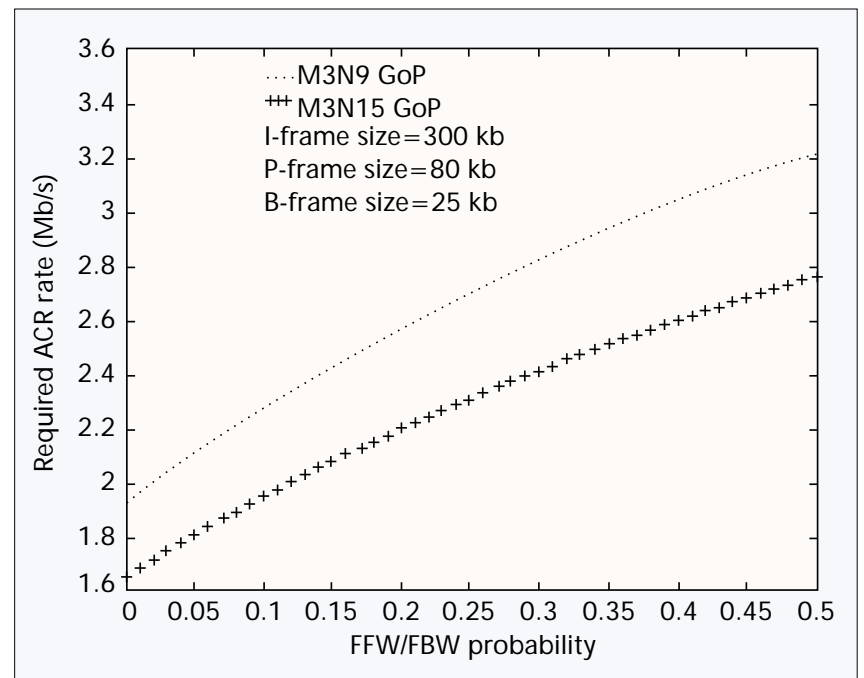

Figure 7. Variation of bandwidth requirement as a function of the level of interactivity of the client.

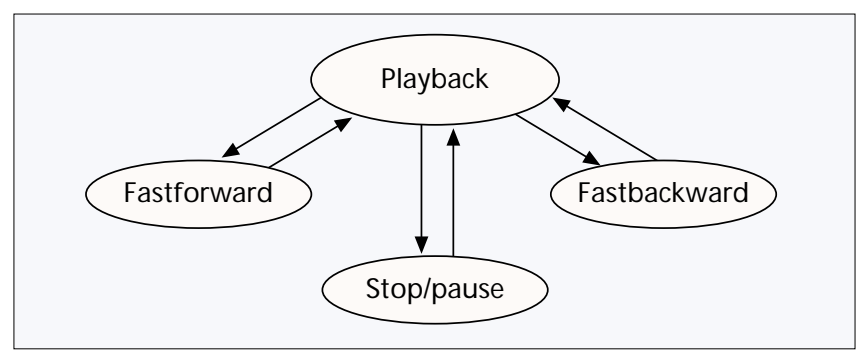

Figure 6. M odeling the interactive client behavior.

need to have $A C R=E[\lambda(t)]$. The required $A C R$ varies with the level of interactivity of the client. Figure 7 shows the variation of the required $A C R$ as a function of the level of client interactivity for a typical M PEG -2 stream.

B ecause the ABR service utilizes the excess bandwidth of the network, congestion in the network will result in a lower bandwidth (ACR) obtained by the server from the network. In this case, any request for a high bandwidth by the server will be rejected with a high probability. The expected ACR from the network as a function of the level of network congestion is shown in Fig. 8 [6].

In the system described above, the use of trick modes by the client is followed by a startup delay required to fill up the client buffer before the commencement of video display. Startup delay is defined as the time between the user pressing Playback (from the Stop state) or F ast Forward/Backward to the start of the video display. The startup delay will depend on the bandwidth allocated to the server. Figure 9 shows the relationship between the startup delay and the PCR, M CR parameters [6]. It shows that the startup delay can be minimized by negotiating a large PCR during connection setup (we assume that the MCR is fixed at the average rate of the video).

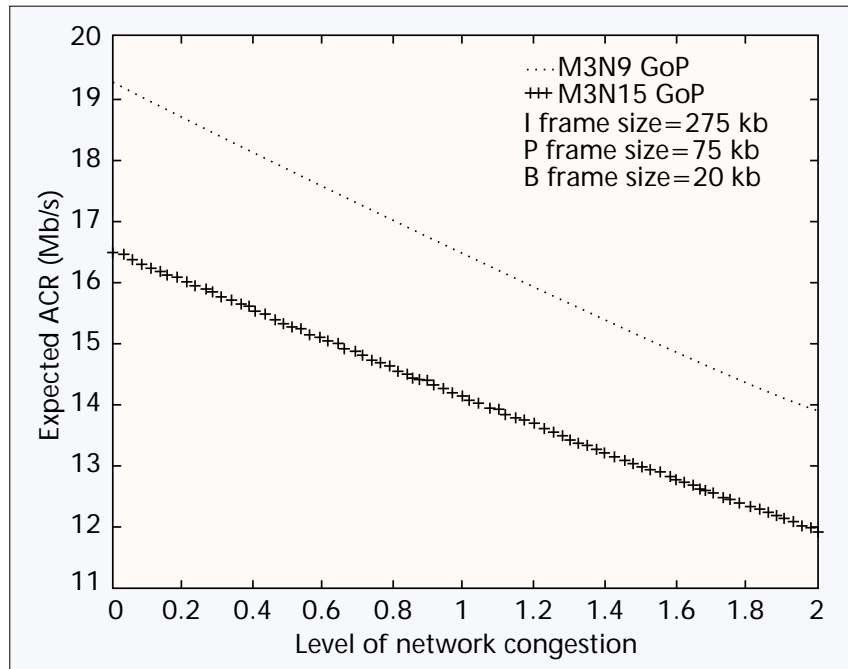

Figure 8. Expected $A C R$ as a function of network congestion for a PCR/MCR ratio of 20 . 


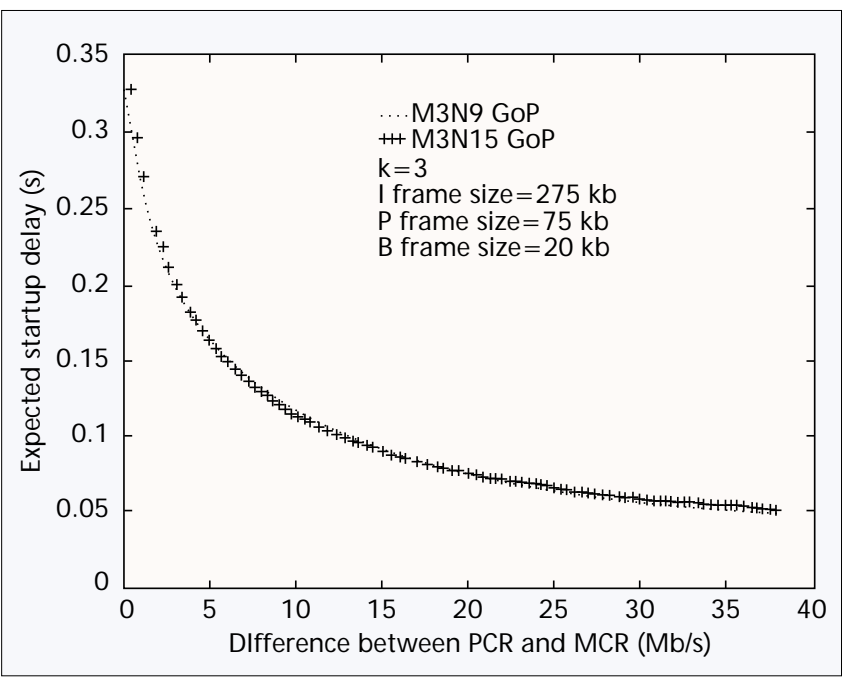

Figure 9. Expected startup delay versus PCR.

\section{Congestion CONTROL}

Congestion is defined as a phenomenon where the amount of traffic injected into the network is more than the capacity of the network, resulting in possible overflow of buffers in the network. Congestion control can be achieved using either open-loop or closed-loop mechanisms. The open-loop scheme is based on designing and configuring the system carefully to avoid the occurrence of congestion. The closed-loop scheme in ATM networks is based on feedback (discussed earlier) from the network to the ABR sources to slow down. ABR sources have traditionally been data traffic. $\mathrm{H}$ owever, studies [7] have shown that multimedia traffic can be efficiently transported over ABR service. Congestion control, therefore, implies that the multimedia sources need to slow down in order to help the network get out of congestion. Congestion control can be implemented at either the end system or the network, as described below.

\section{Congestion Control at the End System}

When congestion control is implemented at the end system, the multimedia server adjusts the video source rate based on the level of network congestion. It is different from rate control at the end system in the sense that congestion control at the end system is aimed at helping the network to recover from congestion, whereas rate control is an end-to-end process.

Feedback from the network can be used by the server as an indication of the level of network congestion. A Iternatively, the buffer occupancy at the server can also be used as a measure of the level of congestion in the network. The buffer occupancy can be used to control the transmission rate from the video server $[7,8]$. Feedback from the server buffer has the advantage of a much smaller delay when compared to the delay in obtaining feedback from the network.

\section{Congestion Control by the Network}

Congestion control can be done at the network level by dropping video cells in the switches [9]. The following two methods could be used by the network to drop video cells.

Random Cell Discarding - In the RCD scheme, the network discards video cells regardless of its importance. A Ithough this method is easy to implement, it may seriously degrade the picture quality if the I-frames are dropped.

Priority Packeting and Selective Cell Discarding - In the PPSCD scheme, video frames are encapsulated into ATM cells with different discard priorities for different frames [9].
Since the network discards low-priority cells first, the degradation in picture quality will be much less than with the R CD scheme. Compared to the R CD scheme, the PPSCD is relatively difficult to implement.

The R CD scheme is easy to implement, but may seriously degrade the quality of multimedia. On the contrary, in the PPSCD scheme the server assigns cell discard priority to the different frames of video in order to guarantee an acceptable Q oS in multimedia transmission.

\section{FLOW CONTROL}

Flow control is a point-to-point traffic control mechanism between the server and client. I t regulates the server into sending no faster than the client can handle. In multimedia transmission over a high-speed A TM network, it is extremely important to maintain the traffic flow at an optimum rate. Too fast a rate will result in buffer overflow (leading to loss of multimedia traffic) at the client. A very slow rate will result in buffer underflow at the client, leading to still frames and interruption in video display at the client.

For a LAN -based multimedia system, flow control can be implemented by the client sending information about its buffer level to the server, as shown in Fig. 10. B ecause the round-trip time (RTT) between the client and server is small and varies over a small range, it is relatively easy to match the server's transmit speed to the dynamic variation of the client buffer level.

The large control latency resulting from a high value of R TT in a distributed multimedia system makes it difficult to implement the scheme based on direct feedback between the client and server. Hop-by-hop flow control can be used to solve the above problem. In the hop-by-hop scheme, each node acts like an active local information generator. L et $\lambda_{i}(t)$ denote the outgoing rate for node $i$ at time $t, X_{i+1}(t)$ the buffer occupancy at node $i+1$ at time $t$, and $\tau$ the time interval for sampling of the buffer occupancy. The sending rate at node $i$ is periodically computed based on the local server capacity and the buffer occupancy of the downstream switching node using $\lambda_{1}(t)=F\left(X_{i+1}(t-\tau), \lambda_{1}(t-\tau)\right)$. This scheme can overcome the long response time between the server and client of a multimedia system. H owever, since each node needs to calculate the sending rate periodically, it requires a large amount of computing power at the nodes of a network with a large number of nodes and connections, eventually giving rise to scalability issues.

\section{RATE-SCALABle Multimedia TRANSMission}

It is important to have continuous transmission of multimedia traffic even during congestion in the network. Rate-scalable transmission refers to scaling the bit rate of multimedia traffic during periods of network congestion with a view to maintaining continuous transmission. However, this is achieved, in most cases, at a graceful degradation of video quality at the client. The following are some of the techniques that can be used to achieve rate-scalable transmission of a multimedia stream.

The Transcoding Scheme - is based on first decoding the compressed video, and then reencoding the video at a lower bit rate [10]. The cost of a real-time hardware encoder unit per multimedia stream makes this method very expensive for realtime multimedia applications. The advantages of this scheme are that it is straightforward, and the bit rate of the encoder can easily be controlled by the feedback from the network.

The Multiple-Rate Coding Scheme - encodes and stores video at a number of different bit rates. All the streams are 
played back simultaneously in a lockstep fashion and sent via a switch to the server, which receives feedback from the network. If the network is congested, the server switches to a lower-bit-rate video stream. This scheme does not require expensive hardware since the encoding can be done offline using a cheap software encoder. It has the disadvantages of requiring large capacity to store the different bit rate versions of the same video. M oreover, simultaneous playback increases the load on the video server. This scheme may also result in low bandwidth utilization since the video is stored in fixed bit rates.

The Frame Dropping Scheme - drops frames in order to match the reduced available bandwidth from the network. In the case of congestion in the network, less important frames may be dropped by the server. The least important B-frames are dropped first, followed by dropping of P-frames if required. If further reduction of bit rate is necessary, complete G oPs may be dropped. The advantages of this scheme are that it does not require additional hardware, and it achieves a high multiplexing gain. This scheme, however, increases the computational complexity of the server because of its requirement to decide on the frames to be dropped and to implement the frame dropping.

In the Block Dropping Scheme - blocks of frames are selectively dropped at the server to reduce the transmission bandwidth requirements [10]. At the client, the dropped blocks are interpolated from the neighboring blocks of the frame.

In Layered Coding - the video is coded into a number of layers, called the base layer and enhancement layers [11]. The enhancement layers, combined with the base layer, increase the quality of the video. In order to reduce the video bit rate during periods of network congestion, the enhancement layers are not transmitted from the server.

\section{SUMmARY}

We review the issues and possible solutions in managing multimedia traffic over A TM networks. We find that although the issues have distinct features and functions, many of them use similar techniques such as feedback. When traffic shaping is implemented by the network, the R andom C ell D iscard scheme is easy to implement, but may degrade the Q oS of multimedia streams. Although the Priority Packeting and Cell Discard scheme is difficult to implement, its selective dropping of cells will help in maintaining an acceptable Q oS of the video stream.

The adaptive capability of feedback-based congestion control makes it suitable for multimedia traffic whose characteristics cannot be fully defined a priori. On the contrary, prediction-based congestion control works well only when an accurate model of the traffic stream is available.

The capability of available bit rate service to request more bandwidth than is currently being used by an ABR source is very suitable for requesting additional bandwidth required during the trick modes of interactive applications.

Direct feedback from the client can be successfully used to regulate the transmission rate of the server in a $L A N$ environment. H owever, for a large-sized distributed multimedia system, the large round-trip delay renders the direct feedback impractical to use. In such a case, the hop-by-hop scheme can be used to offset the effect of large feedback latency at the expense of lowering the utilization of the nodes.

Several schemes to scale the bit rate of the multimedia, such as frame dropping and block dropping, can be used to scale down the bit rate of a precoded multimedia stream during periods of network congestion.

\section{REFERENCES}

[1] B. Vandalore et al., "Quality of service and multipoint support for multimedia applications over atm abr service," IEEE Commun. Mag., this issue.

[2] M. Graf, "VBR video over ATM: Reducing network resource requirement through endsystem traffic shaping," Proc. IEEE INFOCOM '97, Kobe, Japan, Apr. 7-11 1997, pp. 48-57.

[3] M. Krunz and S. K. Tripath, "Exploiting the temporal structure of MPEG2 video for the reduction of bandwidth requirement," Proc. IEEE INFOCOM '97, Kobe, Japan, Apr. 1997, pp. 143-50.

[4] M. Hamdi, J. W. Roberts, and P. Rolin, "Rate control for VBR video coders in broadband networks," IEEE JSAC, vol. 15, no. 6, Aug. 1997, pp. 1040-51.

[5] B. Zheng and M. Atiquzzaman, "Video on demand over ATM: system design and networking requirements," Proc. ENCOM'98, Atlanta, GA, June 7-11, 1998.

[6] B. Zheng and M. Atiquzzaman, "Multimedia over high speed networks: reducing network requirement with fast buffer fillup," Proc. IEEE GLOBECOM '98, Sydney, Australia, Nov. 8-12, 1998.

[7] H. Kanakia, P. P. Mishra, and A. R. Reibman, "An adaptive congestion control scheme for real time packet video transport," IEEE/ACM Trans. Networking, vol. 3, no. 6, Dec. 1995, pp. 671-82.

[8] K. Karahara, Y. O. M. Murata, and H. Miyahara, "Performance analysis of reactive congestion control for ATM network," IEEE JSAC, vol. 13, no. 4, May 1995, pp. 651-61.

[9] A. Mehaoua and R. Boutaba, "Performance analysis of cell discarding techniques for best effort video communications over ATM networks," Comp. Networks and ISDN Sys., vol. 29, no. 17-18, Feb. 1998, pp. 1999-2019.

[10] W. Zeng and B. Lu, "Rate shaping by block dropping for transmission of MPEG precoded video over channels of dynamic bandwidth," Proc. 4th ACM Multimedia Conf., Boston, MA, Nov. 18-20, 1996, pp. 385-93.

[11] G. Seckin and F. Goulshani, "A new model for multilayer real-time video transmission for ATM networks," Proc. 7th Int'l. Conf. Comp. Commun. and Networks, Lafayette, LA, Oct. 12-15,1998, pp. 442-47.

\section{BIOGRAPHIES}

BING ZHENG (zhengbin@flyernet.udayton.edu) is a Ph.D. student working in multimedia and networking at the Department of Electrical and Computer Engineering, University of Dayton. He received a B.Sc. in physics in 1984 and an M.En in electro-optics in 1990. From 1984 to 1987, he was a teaching assistant at West China University of Medical Science. Before joining the Department of ECE of UD in August 1997, he was an associate professor working in integrated optoelectronic technology, optical fiber technology, WDM technology, and flat panel display technology with the University of Electronic Science and Technology of China (UESTC). He has numerous technical publications in these areas. His current research interests include ATM, multimedia, network system design, traffic management, protocol design, and WDM applications.

MOHAMmed ATIQUZZAMAN (atiq@engr.udayton.edu) received M.Sc and Ph.D. degrees in electrical engineering and electronics from the University of Manchester Institute of Science and Technology, U.K. Currently he is a faculty member in the Department of Electrical and Computer Engineering at the University of Dayton, Ohio. He is a senior editor of IEEE Communications Magazine and member of the advisory editorial board of Computer Communications He has guest edited many special issues of various journals including special issues on ATM switching and ATM networks of the International Journal of Computer Systems Science \& Engineering, Next Generation Internet in the European Transactions on Telecommunications and Optical Networks, and Communication Systems and Devices in IEEE Communications Magazine. He has also served on the technical program committees of many national and international conferences, including IEEE INFOCOM and IEEE Annual Conference on Local Computer Networks. His current research interests are in Broadband ISDN and ATM networks, multimedia over high-speed networks, and switching. He has over 90 refereed publications. 